\title{
Relação entre a prática de actividade física e depressão em estudantes universitários
}

\section{Relationship between the practice of physical activity and depression in university students}

ARTIGO ORIGINAL | ORIGINAL TITLE

\begin{abstract}
RESUMO
Os objectivos deste estudo foi comparar por sexo, prática e frequência de actividade física ao nível da depressão global. A amostra deste estudo foi constituída por 183 indivíduos, sendo 38,3\% do sexo masculino e $61,7 \%$ do sexo feminino. Os resultados indicam que são os homens quem mais pratica, com maior frequência e com sessões mais longas de actividade. Constatou-se a existência de efeitos significativos nas comparações por sexo ao nível da depressão global e na dimensão afectivo-somática, assim como há diferenças significativas entre sexos quanto à prática de actividade física e na depressão. Posto isto, concluímos que os homens praticam mais do que as mulheres, e elas tendem a apresentar valores de depressão superiores. As mulheres apresentam níveis mais elevados de depressão em todas as suas dimensões e de forma significativa na dimensão afectivo-somática.
\end{abstract}

Palavras-chave: actividade física, depressão, BDI

\begin{abstract}
This study's objectives were to compare sex, practice, and frequency of physical activity in terms of global depression. This study's sample consisted of 183 individuals, $38.3 \%$ male and $61.7 \%$ female. The results indicate that it is men who practice more frequently and with longer sessions of activity. Comparison by sex showed significant effects in terms of global depression and the somatic-affective dimension. There are significant differences between men and women regarding the practice of physical activity and depression. That said, we conclude that men practice more than women, who tend to have higher values on depressive symptoms. Women have higher levels of depression in all its dimensions, particularly in the affective-somatic dimension.

Keywords: physical activity, depression, BDI
\end{abstract}

Ana Lima. Exercise \& Health Psychologist, Independent Behavioral Consultant. London, UK.

e-mail: anaguida.lima@gmail.com

Anabela Beleza. Mestre em Psicologia do Exercício e Saúde, Formadora. Mondim de Basto, Portugal.

e-mail: beleza.anabela10@gmail.com 
A depressão é uma das patologias com maior prevalência na sociedade actual (Lawlor \& Hopker, 2001; Bahls, 2002; Bonilla, G.Santos, \& D.Santos, 2004), traduzindo-se num grave problema de saúde pública (Üner \& Özcebe, 2008). Segundo Vieira, Porcu e Rocha (2007) e de acordo com a Organização Mundial de Saúde (OMS) a prevalência deste transtorno tem aumentado nos últimos anos, atingindo cerca de $5 \%$ da população mundial.

Esta patologia tende a ser duas vezes superior nas mulheres (Buckworth \& Dishman, 2002; Kuritza \& Gonçalves, 2003; Galambos, Leadbeater, \& Barker, 2004; Justo \& Calil, 2006; VieIra et al., 2007; Leach, Christensen, Makinnon, Windsor, \& Buterworth, 2008), contudo esta diferença entre os sexos não é estável ao longo da idade, pois tende a ser mais acentuada na adolescência e na adultícia, diminuindo na terceira idade (Leach et al., 2008).

Apesar dos avanços científicos e clínicos no âmbito da saúde mental, a compreensão plena da depressão assim como o seu tratamento continuam incerto. No âmbito da intervenção clínica há mesmo uma busca intensa por formas alternativas à psicofarmacologia. Alguns avanços têm evidenciado a relevância que o exercício físico poderá ter como forma de tratamento. Tendo em conta esta associação, considerámos pertinente esclarecer as diferenças entre os conceitos de actividade física e exercício, que apesar de distintos se encontram inter-relacionados, por isso, ao longo deste trabalho serão usados de forma indiscriminada. Podemos definir actividade física como o movimento produzido pelos músculos esqueléticos, do qual resulta dispêndio energético (Buckworth \& Dishman, 2002). O exercício físico é uma "subcategoria da actividade física, é planeada, estruturada, engloba os movimentos corporais repetitivos de uma pessoa que tem como propósito melhorar ou manter componentes físicas ou de saúde" (idem, p. 28).

A depressão é um transtorno de humor que pode ser entendida por manifestações afectivas anómalas que oscilam quanto à intensidade, frequência e duração dos sintomas (Vieira et al., 2007). Assim, e de acordo com Manual de Diagnóstico e Estatística das Perturbações Mentais (APA, DMS-5, 2013), a depressão caracteriza-se pelo humor depressivo, diminuição do interesse, perda de peso, distúrbios no sono, fadiga ou perda de energia e sentimentos de desvalorização ou culpa. Os indivíduos com depressão deixam de se interessar por actividades que até então lhes eram agradáveis, a sua vida perde o sentido, sentem-se constantemente tristes, tendem a interiorizar os aspectos negativos e a afastarem-se dos positivos, isolam-se no seu próprio mundo que o constroem com uma visão que é exterior a eles, negativa e distorcida, e quem os leva a entrar em desespero ou tornar-se apáticos e com pensamentos relacionados com a morte (Kuritza \& Gonçalves, 2003). A gravidade da depressão é determinada pelo desajuste do indivíduo com a realidade, podendo ainda ser classificada de diferentes maneiras: depressão leve, em que o transtorno existe, mas não afecta significativamente o funcionamento do indivíduo; depressão moderada, em que já se verifica um comprometimento do funcionamento ao nível da sua produção e percepção; e, depressão grave, onde se verifica a quebra de relações com tudo e com todos, isolamento e, em casos extremos, pode levar à morte.

A depressão pode surgir como consequência de um conflito interno e de alterações bioquímicas, bem como, de problemas psicológicos e sociais (Kuritza \& Gonçalves, 2003; Üner \& Özcebe, 2008). Esta patologia está ainda associada ao stresse e ansiedade (Buckworth \& Dishman, 2002; Saluja et al., 2004; Keenan-Miller, Hammen, \& Brennan, 2007), a perturbações de atenção e comportamento (Saluja et al., 2004), acontecimentos de vida adversos (Buckworth \& Dishman, 2002; Keenan-Miller et al., 2007; Keller, Neale, \& Kendler, 2007), ausência de auto-cuidado, 
ambientes mal adaptativos (Keenan-Miller et al., 2007), fracas relações parentais e de pares (Field, Diego, \& Sanders, 2001) e consumo de substâncias (Saluja et al., 2004).

A actividade física tem sido cada vez mais alvo de estudo devido aos seus efeitos terapêuticos na depressão (Lawlor \& Hopker, 2001). Actualmente, tem surgido um conjunto de estudos sobre a implementação de tratamentos não farmacológicos, nomeadamente a actividade física, que tem demonstrado efeitos anti-depressivos em indivíduos com depressão leve e moderada (Paluska \& Schwenk, 2000; Kuritza \& Gonçalves, 2003; Nabkasorn et al., 2005). A actividade física surge então como uma alternativa, quer para o tratamento, quer para a prevenção de doenças mentais e na melhoria dos estados de humor, nomeadamente na depressão (Werneck, Filho, \& Ribeiro, 2006). A actividade física mostra-se mais efectiva quando combinada com a psicoterapia do que por si só (Machado \& Mota, 1996; Paluska \& Schwenk, 2000; Nabkasorn et al., 2005).

De acordo com Richardson et al. (2005), quando utilizamos a actividade física como complemento do tratamento da depressão, podemos dividi-la em dois tipos: a actividade física estruturada e o estilo de vida activo. O primeiro tipo garante níveis de actividade seguros e mais apelativos num cenário de supervisão, porém, tem como principal desvantagem o custo do espaço, equipamento e acompanhamento. Já o segundo é de baixo custo, flexível e de fácil integração na planificação do dia-a-dia, como é o caso da caminhada, que quase toda a gente pode realizar no tempo de lazer.

As evidências acumulam-se sobre o impacto da actividade física na redução dos sintomas depressivos. Na literatura da especialidade surgem diferentes hipóteses que ilustram os efeitos positivos da actividade física nos sintomas depressivos. A primeira refere-se à interacção social que resulta das relações esta- belecidas durante a prática de actividade física (Machado \& Mota, 1996; Paluska \& Schwenk, 2000; Kuritza \& Gonçalves, 2003; Richardson et al., 2005). Outra das hipóteses apresentadas refere-se à distracção ou time out, que assenta na ideia de que a actividade física funciona como uma abstracção de estímulos stressantes e sentimentos adversos (Machado \& Mota, 1996; Paluska \& Schwenk, 2000; Daley, 2002). A hipótese da mestria e do auto-controlo também surge como um possível efeito da actividade física, na medida em que esta aumenta a capacidade física e funcional do indivíduo (Machado \& Mota, 1996; Paluska \& Schwenk, 2000; Daley, 2002; Kuritza \& Gonçalves, 2003). Destaca-se ainda a hipótese de natureza bioquímica, que está relacionada com a libertação de monoaminas e endorfinas, responsáveis pelo aumento das transmissões sinápticas e dos afectos positivos respectivamente (Machado \& Mota, 1996; Paluska \& Schwenk, 2000; Daley, 2002; Kuritza \& Gonçalves, 2003), bem como o aumento dos baixos níveis de norepinefrina que estão associados à depressão (Machado \& Mota, 1996). Por último, evidencia-se a hipótese termogénica, que se baseia no aumento da temperatura corporal causado pela prática de actividade física e associado à redução de tensão muscular, e, por sua vez, conduz a uma melhoria nos estados psicológicos (Paluska \& Schwenk, 2000; Daley, 2002). Outra possível explicação destes efeitos argumenta com base no facto de a actividade física provocar informação proprioceptiva contraditória ao esquema cognitivo-emocional da depressão (Mota \& Machado, 1996). Para além destes efeitos, a prática de actividade física apresenta outras vantagens quando comparada com outros meios de intervenção, como ser mais saudável e mais acessível a nível económico (Cooper- Patrick, Ford, Mead, Chang, \& Klag, 1997; Richardson et al., 2005; Werneck et al., 2006).

Constatou-se que quer a actividade física 
aeróbia quer a anaeróbia tem efeitos na redução dos sintomas depressivos (Paluska \& Schwenk, 2000; Werneck et al., 2006), contudo é a actividade física contínua, regular, não competitiva e de intensidade moderada (entre 20 a 40 minutos) que produz maior efeito e cria mais aderência (Cooper- Patrick et al., 1997; Paluska \& Schwenk, 2000; Richardson et al., 2005; Werneck et al., 2006). Apesar destas recomendações, a actividade física deve ser adaptada a cada praticante de forma individual (Werneck et al., 2006), tendo em conta o objectivo com que se pratica, a idade, o sexo, o estatuto socioeconómico, a cultura, a forma física, o estado de saúde, as barreiras e os limites à prática de actividade física (Richardson et al., 2005). Segundo Werneck et al. (2006), a prática deve ocorrer em locais agradáveis, proporcionar prazer ao praticante, evitando grandes esforços de modo a que o indivíduo se sinta bem no final. De acordo com Machado e Mota (1996) e numa meta-análise realizada por North, McCullagh e Tran verificou-se que a actividade física produz efeitos na redução da depressão, tanto em populações clínicas como não clínicas. Contudo, Kuritza e Gonçalves (2003) referem que em indivíduos com depressão leve/moderada o exercício aeróbio apresenta maiores efeitos anti-depressivos, enquanto que, em depressões graves/ profundas este tipo de exercício apenas proporciona uma melhoria no seu estado físico, não alterando a severidade da doença.

No nosso estudo, optámos por uma população de estudantes universitários, pois, para além de ser uma população mais acessível, estes estão ainda mais vulneráveis, visto que nesta etapa da vida são necessários alguns ajustes em situações stressantes específicas, resultantes da vida académica (Rezende, Abrão, Coelho, \& Paços, 2008), nomeadamente a pressão da entrada na universidade, preocupação com o futuro e com o desemprego (Üner \& Özcebe, 2008).

Tendo em consideração que iremos estudar indivíduos saudáveis debruçar-nos-emos fundamentalmente na identificação dos sintomas depressivos globais. Apenas quando comparamos os estudantes por sexo é que as variáveis dependentes foram, em acréscimo a depressão global, as dimensões afectivo-somática e auto-depreciação. Assim, os objectivos específicos foram: comparar por sexo, prática (ou não), frequência semanas e tempo de actividade física ao nível da depressão global.

\section{MÉTODOS}

\section{Amostra}

A amostra deste estudo foi constituída por 183 indivíduos $(n=183)$, sendo $38.3 \%$ do sexo masculino $(n=70)$ e $61.7 \%$ do sexo feminino $(n=113)$. A idade dos sujeitos está compreendida entre os 17 e os 32 anos, com uma média de 20.75. Relativamente ao estado civil, a amostra é predominantemente constituída por solteiros $(n=180)$ sendo duas mulheres e um homem casados. Quanto à prática de actividade física, $62.8 \%$ são praticantes $(n=115) \mathrm{e}$ $37.2 \%$ não tem qualquer tipo de actividade ( $n$ $=68$ ) sendo que destes $88.24 \%$ são mulheres. No que diz respeito ao tipo de actividade física, $13.7 \%$ envolvem-se em actividades individuais $(n=25), 31.1 \%$ em actividades colectivas ( $n$ $=57), 8.2 \%$ em actividades de academia ( $n$ $=15)$ e $9.8 \%$ várias modalidades $(n=18)$. A maioria das mulheres não participa $(n=59$, e as activais participam maioritariamente em actividades desportivas quer sejam individuas $(n=21)$ ou colectivas $(n=23)$. Ao nível da frequência, $7.1 \%$ realizam actividades uma vez por semana ( $n=13 ; \mathrm{H}=6, \mathrm{M}=7$ ), $30.1 \%$ duas a três vezes $(n=55 ; \mathrm{H}=28, \mathrm{M}=27)$, $17.5 \%$ três a cinco vezes $(n=32 ; \mathrm{H}=17, \mathrm{M}=$ 15) e $8.2 \%$ mais de cinco vezes $(n=15 ; \mathrm{H}=$ 11; $M=4)$. Por fim, e relativamente à duração, $0.5 \%$ refere praticar menos de 30 minutos ( $n$ $=1), 7.1 \%$ pratica entre 30 a 40 minutos $(n=$ 13), $14.8 \%$ mencionam a prática durante uma hora $(n=27)$ e $40.4 \%$ pratica mais de uma hora $(n=74 ; \mathrm{H}=48 . \mathrm{M}=26)$. 
A técnica de amostragem foi a de conveniência. Não se verificou o abandono de qualquer participante nem a ocorrência de qualquer evento que pudesse ter influenciado o preenchimento dos questionários.

\section{Instrumentos}

O Inventário de Depressão de Beck (BDI) foi desenvolvido por Beck et al. em 1961 e é composto por 21 categorias de sintomas que descrevem uma manifestação comportamental específica da depressão (Beck et al., 1961; Beck, 1967; Byrne \& Baron, \& Campbell, 1993; Gorenstein \& Andrade, 1998). Este inventário foi validado para a língua portuguesa por Wang, Andrade e Gorenstein (2005). Cada item é constituído por 4 afirmações que descrevem 4 possibilidades, ordenadas pela gravidade dos sintomas e cotadas de 0 a 3 variando entre o neutro e o máximo (Beck et al., 1961; Beck, 1967; Byrne \& Baron, 1993; Gorenstein \& Andrade, 1998). O sujeito deve escolher em cada item a opção que melhor descreve a forma como se tem sentido na última semana (Byrne \& Baron, 1993). Os itens do questionário referem-se a uma determinada atitude ou sintoma específico. Beck et al. (1961) referem: tristeza, pessimismo, sensação de fracasso, insatisfação, sentimento de culpa, sensação de punição, auto-depreciação, auto-acusação, ideação suicida, choro, irritabilidade, retracção social, indecisão, distorção da imagem corporal, inibição para o trabalho, distúrbios de sono, fadiga, perda de apetite, perda de peso, preocupação somática e perda de interesse sexual.

Tendo em conta o propósito do nosso estudo, a nossa variável dependente é a depressão com as dimensões afetivo-somática e auto-depreciação. As variáveis independentes são: sexo, prática, frequência e duração de actividade física.

\section{Procedimentos}

O questionário foi aplicado a estudantes de diferentes cursos de uma instituição de ensino superior no norte de Portugal. Foi dada ao participante uma pequena instrução acerca da temática e do preenchimento do questionário, chamando à atenção para não deixarem nenhuma questão por responder. Todos os participantes assinaram um consentimento informado onde era afirmado que poderiam desistir a qualquer momento.

Para procedermos à introdução dos dados e posterior análise estatística utilizámos o software Statistical Package of Social Sciences 26 (SPSS). Para a caracterização da amostra realizámos a análise descritiva e de frequências, e para verificar se existem diferenças entre os sexos relativamente à prática de actividade física foi realizado o teste de Qui-quadrado $\left(\chi^{2}\right)$. Outro procedimento efectuado foi a one-way MANOVA, com o intuito de verificar o efeito das variáveis sexo, prática, frequência e duração de actividade física na depressão. Tendo em consideração a desigualdade entre o número de participantes por cada variável independente em causa, recorremos ao teste de Trace-Pillai.

\section{RESULTADOS}

Relativamente à prática de actividade física por sexos, verificou-se que $88,6 \%$ dos que praticam são do sexo masculino $(n=62)$, enquanto que $46.9 \%$ são do sexo feminino ( $n=53$ ); quanto aos não-praticantes $11.4 \%$ pertencem ao sexo masculino $(n=8)$ e $53.1 \%$ ao sexo feminino $(n=60)$. Deste modo, podemos dizer que as diferenças entre sexos se mostraram significativas $\left(\chi^{2}=32.140 ; p=<.001\right)$ no que diz respeito à prática de actividade física.

Os resultados da one-way MANOVA, para a variável sexo, não evidenciaram qualquer efeito significativo (Pillai $(3,179)=1.732, p=.062$, eta-square $=.018)$. Porém a análise univariada demonstrou um efeito pequeno nas variáveis depressão global e afectivo-somática, A análise univarida apresentou efeitos pequenos nas variáveis depressão global e afectivo somática, como pode ser observado no Quadro 1. 
Quadro 1

Análise univariada para sexo ao nível da depressão global, afectivo-somática e auto-depreciação.

\begin{tabular}{|c|c|c|c|c|c|}
\hline & $\begin{array}{c}\text { Sexo feminino } \\
\mathrm{M} \pm \mathrm{DP}\end{array}$ & $\begin{array}{c}\text { Sexo masculino } \\
\mathrm{M} \pm \mathrm{DP}\end{array}$ & $\mathrm{F}$ & $p$ & $\begin{array}{c}\text { Eta } \\
\text { squares }\end{array}$ \\
\hline Depresão global & $6.938 \pm 5.819$ & $5.129 \pm 5.250$ & 4.498 & .035 & .024 \\
\hline Dim.afectivo-somática & $4.071 \pm 3.833$ & $2.871 \pm 3.002$ & 4.964 & .027 & .027 \\
\hline Dim. auto-depreciação & $1.912 \pm 2.115$ & $1.571 \pm 2.004$ & 1.163 & .282 & .006 \\
\hline
\end{tabular}

Comparação por prática de actividade física evidenciou não existirem diferenças (Pillai (3, 179) $=.018, p=.345$, eta-square $=.018)$. Ao avaliarmos o efeito da prática de actividade física na depressão global podemos constatar que quem pratica tem valores mais baixos ao nível da depressão global embora não sejam significativos, e o efeito estatístico é baixo.

Quadro 2

Efeito da variável prática de actividade física na depressão geral.

\begin{tabular}{cccccc}
\cline { 2 - 5 } & $\begin{array}{c}\text { Praticantes } \\
\mathrm{M} \pm \mathrm{DP}\end{array}$ & $\begin{array}{c}\text { Não-praticantes } \\
\mathrm{M} \pm \mathrm{DP}\end{array}$ & $\mathrm{F}$ & $p$ & $\begin{array}{c}\text { Eta } \\
\text { squares }\end{array}$ \\
\hline Depresão global & $5.670 \pm 5.241$ & $7.221 \pm 6.231$ & 3.245 & .073 & .018 \\
Dim.afectivo-somática & $3.252 \pm 3.211$ & $4.221 \pm 4.033$ & 3.168 & .077 & .017 \\
Dim. auto-depreciação & $1.643 \pm 1.802$ & $2.015 \pm 2.465$ & 1.371 & .243 & .008 \\
\hline
\end{tabular}

As comparações por nível de frequência também não produziu qualquer efeito estatístico (Pillai $(12,534)=.064, p=.482$, eta-square $=.21)$. A análise univariada evidenciou existir um efeito moderado ao nível da depressão global $(\mathrm{F}=2.179, p=.071$, eta-square $=.047, \mathrm{PO}=.639)$. Relativamente às dimensões verificaram-se os seguintes valores: efectivo-somática ( $\mathrm{F}=1.949, p=.104)$ e auto-depreciação $(\mathrm{F}=1.399, p=.199)$.

\section{DISCUSSÃO}

Para os efeitos deste trabalho foram definidos como objectivos diferenciar homens e mulheres ao da prática da actividade física. Os nossos resultados apontam para um maior nível de prática de actividade física nos elementos do sexo masculino, o que é corroborado pelos estudos de Camões e Lopes (2008) e Leach et al. (2008). Verificámos, ainda, que são os indivíduos do sexo feminino que apresentam valores mais elevados na depressão geral, embora esta diferença não seja significativa. Apesar da sua não significância, estas diferenças vão de encontro aos achados de Byrne et al. (1993), Gorenstein e Andrade (1998), Galambos et al. (2004), Leach et al. (2008) e Rezende et al. (2008). As análises demonstraram ainda que os praticantes de actividade física apresentam menores médias nos de sintomas de depressão. No entanto, as diferenças de não foram significativas. Estes resultados são partilhados por vários autores: Lawlor e Hopker (2001) que defendem que os praticantes pontuam menos nas escalas da BDI. Estes resultados são coerentes com os de outros estudos que mesmo que com abordagens e objectivos diferentes contribuem para esclarecer os efeitos da actividade física na redução dos sintomas.

Por exemplo, segundo Nabkasorn et al. (2005) as actividades como o jogging causam uma diminuição nos índices de depressão. Por sua vez, Babyak et al. (2000) concluiu que pacientes com depressão que se envolvem na prática de actividade física mostram uma significativa redução dos sintomas depressivos, e esses efeitos tendem a prolongar-se durante seis meses. Brown, Ford, Burton, Marshall e 
Dobson (2005) constataram que existe uma associação significativa e negativa entre prática de actividade física e sintomas depressivos. Segundo Goodwin (2003) esta associação pode também ser explicada de forma inversa, isto é, o transtorno mental pode funcionar como uma barreira à prática de actividade física, sendo que as pessoas deprimidas estão menos disponíveis para essa prática devido à apatia e falta de energia. Deste modo, é difícil estabelecer uma causalidade, no sentido em que, o aumento dos sintomas depressivos pode levar ao declínio da prática de actividade física, isto é, o estado depressivo está associado à inactividade física e ao sedentarismo (Nabkasorn et al., 2005).

Nas suas pesquisas, Mello, Boscolo, Esteves e Tufik (2005) demonstraram que a prática regular de exercício está relacionada com uma menor prevalência de estados depressivos. Goodwin (2003) verificou no seu estudo que a prática regular de actividade física está significativamente relacionada com uma menor probabilidade de vir a desenvolver depressão. Estes resultados relativos à frequência de actividade física vão de encontro aos resultados obtidos no nosso estudo, contudo, os nossos não têm significância estatística. Quando nos referimos à duração da actividade física na depressão, podemos dizer que apesar de não existir um efeito significativo, os níveis de depressão tendem a baixar quando a actividade é superior a uma hora.

Apesar de ser consensual, e de os nossos resultados apontarem para a prática de exercício físico como um factor importante na redução da depressão, ainda não está bem definido como tal ocorre. Assim, é necessário conhecer e compreender qual a intensidade, frequência e duração mais apropriadas para que se verifiquem efeitos efectivos na redução dos sintomas.

\section{CONCLUSÃO}

Após a análise dos nossos dados, verificámos que os sujeitos do sexo feminino apresentam menores níveis de prática de actividade física, bem como, maiores índices de depressão, o que nos permite concluir que a sua baixa prática de actividade poderá estar associada a níveis mais altos de sintomas de depressão ou que na ausência da prática não beneficiam dos efeitos preventivos do exercício físico. Relativamente à frequência da prática de actividade física, podemos dizer que quem pratica mais vezes tende a apresentar menos sintomas depressivos. Para além do facto do indivíduo praticar actividade física temos de ter em conta o tempo da sua prática, pois os efeitos tendem a ser mais visíveis quando esta ocorre naqueles que praticam durante mais de uma hora.

Deste modo, e uma vez que os sintomas depressivos são muitas vezes frequentes e crónicos, devemos apostar na promoção da actividade física como uma técnica eficaz e de baixo custo quer na prevenção quer no tratamento da depressão. Uma das limitações que podemos apresentar neste estudo está relacionado com a dimensão da amostra e por ter considerado estudantes universitários. Seria da maior importância incluir, para comparações, indivíduos clinicamente diagnosticados com depressão e estudar retrospectivamente a sua história de prática de exercício físico.

\section{Agradecimentos:}

Nada declarado.

\section{Conflito de Interesses:}

Nada declarado.

\section{Financiamento:}

Nada declarado.

\section{REFERÊNCIAS}

Associação Americana de Psiquiatria (2013). Manual de Diagnóstico e Estatística das Perturbações Mentais (DSM-5 ${ }^{\mathrm{TM}}$ ). Lisboa: Climepsi Editores.

Babyak, M., Blumenthal, J.A., Herman, S., Khatri, P., Doraiswamy, M., Moore, K., Craighead, 
W.E., Baldewicz, T.T., Krishnan, K.R. (2000). Exercise treatment for major depression: maintenance of therapeutic benefit at 10 months. Psychosomatic Medicine, 62(5):633638. doi: 10.1097/00006842-20000900000006.

Bahls, S. (2002). Aspectos clínicos da depressão em crianças e adolescentes. Jornal de Pediatria, 78(5), 359-366. Doi: 10.1590/S002175572002000500004 .

Beck, A. (1967). Depression: Causes and treatment. Philadelphia: University of Pennsylvania Press.

Beck, A., Ward, C., Mendelson, M., Mock, J., \& Erbaugh, J. (1961). An inventory for measuring depression. Archives of General Psychiatry, 4, 561-571.

Bonilla, J., Santos, G., \& Santos, D. (2004). A revised spanish version of the Beck Depression Inventory: psychometric properties with a Puerto Rican sample of college students. Journal of Clinical Psychology, 60(1), 119-130. Doi: 10.1002/jclp.10195

Brown, W., Ford, J., Burton, N., Marshall, A., \& Dobson, A. (2005). Prospective study of physical activity and depressive symptoms in middle-aged womam. American Journal of Preventive Medicine, 29(4), 265-272. DOI: 10.1016/j.amepre.2005.06.009

Buckworth, J. \& Dishman, R. (2002). Exercise Psychology. Illinois: Human Kinetics.

Byrne, B. \& Baron, P. (1993). The Beck Depression Inventory: testing and cross validating a hierarchical factor structure for non-clinic adolescents. Measuring and Evaluation in Counseling and Development, 26, 164-179. Doi: 10.1016/0005-7967(94) E0050-S

Byrne, B. M., Baron, P., \& Campbell, T. L. (1993). Measuring Adolescent Depression: Factorial Validity and Invariance of the Beck Depression Inventory Across Gender. Journal of Research on Adolescence, 3(2), 127-143. doi:10.1207/s15327795jra0302_2.

Camões, M. \& Lopes, C. (2008). Factores associados à actividade física na população portuguesa. Revista de saúde Pública, 42(2), 208-216. Doi: 10.1590/S003489102008000200004.

Cooper-Patrick, L., Ford, D., Mead, L., Chang, P., \& Klag, M. (1997). Exercise and depression in midlife. American Journal of Public Health, 87(4), 670-673. doi: 10.2105/ajph.87.4.670

Daley, A. (2002). Exercise therapy and mental health in clinical population: is exercise therapy a world while intervention? Advances en Psychiatric Treatment, 8, 262-270. Doi: 10.1192/apt.8.4.262

Field, F., Diego, M., \& Sanders, C. (2001). Adolescent depression and risk factors. Adolescence, 36(143), 491-498. PMID: 11817630

Galambos, N., Leadbeater, B., \& Barker, E. (2004). Gender differences in and risk factors for depression in adolescence: a 4-year longitudinal study. International Journal of Behavior Development, 28(1), 16-25. Doi: 10.1080/01650250344000235

Goodwin, R. (2003). Association between physical activity and mental disorders among adults in the United States. Preventive Medicine, 36, 698-703. DOI: 10.1016/s00917435(03)00042-2

Gorenstein, C. \& Andrade, L. (1998). Inventário de depressão de Beck: propriedades psicométricas da versão em português. Revista de Psiquiatria Clínica, 25(5), 245-250.

Justo, L. \& Calil, H. (2006). Depressão: o mesmo acometimento para homens e mulheres? Revista de Psiquiatria Clínica, 33(2), 74-79. DOI: 10.1590/S0101-60832006000200007

Keenan-Miller, D., Hammen, C., \& Brennan, P. (2007). Health outcomes related to early adolescent depression. Journal of Adolescent Health, 41, 256-262. DOI: 10.1016/j. jadohealth.2007.03.015

Keller, M., Neale, M., \& Kendler, K. (2007). Association of different adverse life events with distinct patterns of depression symptoms. American Journal of Psychiatry, 
164, 1521-1529. DOI: 10.1176/appi. ajp.2007.06091564

Kuritza, A. \& Gonçalves, A. (2003). Actividade física orientada para pacientes depressivos: lançando um novo olhar sobre o corpo. Boletim de Saúde, 17(1), 39-52.

Lawlor DA, Hopker SW. The effectiveness of exercise as an intervention in the management of depression: systematic review and meta-regression analysis of randomised controlled trials. BMJ. 2001 Mar 31; 322(7289):763-7. doi: 10.1136/ bmj.322.7289.763.

Leach, L., Christensen, H., Mackinnon, A., Windsor, T., \& Butterworth, P. (2008). Gender differences in depression and anxiety across the adult lifespan: the role of psicosocial mediators. Social Psychiatry and Psychiatric Epidemiology, 43, 983-998. DOI: 10.1007/s00127-008-0388-z

Machado, J. \& Mota, M. (1996). Efeitos e benefícios psicológicos do exercício e da actividade física. In Cruz, J. (Eds). Manual de Psicologia do Desporto (pp.91-115). Braga: Sistemas Humanos e Organizacionais Lda.

Mello, M., Boscolo, R., Esteves, A., \& Tufik, S. (2005). O exercício físico e os aspectos psicobiológicos. Revista Brasileira de Medicina do Esporte, 11(3), 203-207. Doi: 10.1590/ S1517-86922005000300010.

Nabkasorn, C., Miyai, N., Sootmongkol, A., Junprasert, S., Yamamoto, H., ... \& Miyashita, K.. (2005). Effects of physical exercise on depression, neuroendocrine stress hormones and physiological fitness in adolescent females with depressive symptoms. European Journal of Public Health, 16(2), 179-184. DOI: 10.1093/eurpub/cki159

Paluska, S. A., \& Schwenk, T. L. (2000). Physical Activity and Mental Health. Sports Medicine, 29(3), 167-180. doi:10.2165/00007256-
200029030-00003

Rezende, C., Abrão, C., Coelho, E., \& Passos, L. (2008). Prevalência de sintomas depressivos entre estudantes de medicina da Universidade Federal de Uberlândia. Revista Brasileira de Educação Médica, 32(3), 315-323. DOI: $10.1590 /$ S0100-55022008000300006

Richardson, C., Faulkner, G., McDevitt, J., Skrinar, G., Hutchinson, D., \& Piette, J. (2005). Integrating physical activity into mental health services for persons with serious mental illness. Psychiatric Services, 56(3), 324-331. DOI: 10.1176/appi. ps.56.3.324

Saluja. G., Iachan, R., Scheidt, P., Overpeck, M., Sun, W., \& Giedd, J. (2004). Prevalence of and risk factors for depressive symptoms among young adolescents. Archives of Pediatric and Adolescence Medicine, 158, 760-765. doi:10.1001/archpedi.158.8.760

Ünner, S. \& Özcebe, H. (2008). Assessment of depression and risk factors using Beck Depression Inventory in high-school students. Erciyes Medical Journal, 30(4), 350-358.

Vieira, J., Porcu, M., \& Rocha, P. (2007). A prática de exercícios físicos regulares com terapia complementar ao tratamento de mulheres com depressão. Jornal Brasileiro de Psiquiatria, 56(1), 23-28. Doi: 10.1590/ S0047-20852007000100007.

Wang, Y., Andrade, L., \& Gorenstein, C. (2005). Validation of the Beck Depressive Inventory for a portuguese-speaking Chinese community in Brasil. Brasilian Journal of Medical and Biological Research, 38, 399-408. Doi: 10.1590/S0100-879X2005000300011

Werneck, F., Filho, M., \& Ribeiro, L. (2006). Efeitos do exercício sobre os estados de humor: uma revisão. Revista Brasileira de Psicologia de Esporte e do Exercício, 0, 22-54. 